\title{
A single dose of mirtazapine attenuates neural responses to self-referential processing
}

\section{Komulainen, Emma}

2016-01

Komulainen, E, Heikkila , R, Meskanen , K, Raij , T T, Nummenmaa , L, Lahti , J , Jylhä , P , Melartin , T , Harmer , C J , Isometsa , E \& Ekelund , J 2016 , ' A single dose of mirtazapine attenuates neural responses to self-referential processing ' , Journal of Psychopharmacology, vol. 30 , no. 1 , pp. 23-32 . https://doi.org/10.1177/0269881115616384

http://hdl.handle.net/10138/223848

https://doi.org/10.1177/0269881115616384

publishedVersion

Downloaded from Helda, University of Helsinki institutional repository.

This is an electronic reprint of the original article.

This reprint may differ from the original in pagination and typographic detail.

Please cite the original version. 


\title{
A single dose of mirtazapine attenuates neural responses to self-referential processing
}

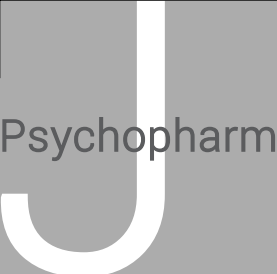

Journal of Psychopharmacology 2016, Vol. 30(1) 23-32 (c) The Author(s) 2015 Reprints and permissions: sagepub.co.uk/journalsPermissions.nav DOI: $10.1177 / 0269881115616384$ jop.sagepub.com

\author{
Emma Komulainen ${ }^{1}$, Roope Heikkilä ${ }^{1}$, Katarina Meskanen ${ }^{1}$, \\ Tuukka T Raij ${ }^{1,2,3}$, Lauri Nummenmaa ${ }^{3,4}$, Jari Lahti ${ }^{5,6}$, Pekka Jylhä ${ }^{1,7}$, \\ Tarja Melartin ${ }^{1}$, Catherine J Harmer ${ }^{8}$, Erkki Isometsä ${ }^{1,7}$ \\ and Jesper Ekelund ${ }^{1,9}$
}

(\$)SAGE

\begin{abstract}
Increased self-focus is a core factor in the psychopathology of depression. Cortical midline structures (CMS) are implicated in the neurobiology of self, depression and antidepressant treatment response. Mirtazapine, an antidepressant that increases serotonin and norepinephrine release, enhances processing of positive and attenuates processing of negative emotional information in healthy volunteers after a single dose. These early changes, which are opposite to the negative information bias in depression, may be important for the therapeutic effect of mirtazapine. It nevertheless remains unresolved whether/how mirtazapine specifically influences processing of self-referential emotional information.

Half of the healthy volunteers $(n=15 / 30)$ received a single dose of mirtazapine, in an open-label design, two hours before functional magnetic resonance imaging (fMRI), and the other half was scanned as a control group without medication. During fMRI the participants categorized positive and negative self-referential adjectives.

Mirtazapine attenuated responses to self-referential processing in the medial prefrontal cortex and the anterior cingulate cortex. Mirtazapine further decreased responses to positive self-referential processing in the posterior cingulate cortex and parietal cortex.

These decreased responses of the CMS suggest that mirtazapine may rapidly improve the ability of the CMS to down-regulate self-referential processing. In depressed patients, this could lead to decreased self-focus and rumination, contributing to the antidepressant effect.
\end{abstract}

Keywords

Antidepressant, mirtazapine, fMRI, emotions, self-referential processing

\section{Introduction}

Cognitive theories of depression propose that biases in cognitive processes play a key role in the pathogenesis of the depression (Gotlib and Joormann, 2010). Depressed patients pay more attention to negative events and have difficulties in recognizing subtle positive emotions and inhibiting negative information (Gotlib and Joormann, 2010). Increased negative thoughts about self are also characteristic of depression (Northoff, 2007). Depression is associated with an increased level of self-focus, rather than paying attention to the external environment (Northoff, 2007), and this is related to an increased experience of negative affect (Mor and Winquist, 2002). Self-blame and feelings of worthlessness are well-known symptoms of depression, and the self-blame factor is associated with a higher number of suicide attempts (Grunebaum et al., 2005). Ruminative response style is closely related to increased self-focus, as it is defined as an individual's focus of attention to his or her negative emotional state (NolenHoeksema, 1991). Rumination has been found to predict higher levels of depression symptoms and longer episodes of depressed mood in depressed patients (Nolen-Hoeksema, 1991).

Neuroimaging research has consistently shown that selfreferential processing is associated with activity of the cortical midline structures (CMS), particularly the medial prefrontal cortex
(MPFC), but also the anterior and posterior cingulate cortex (ACC and PCC), the precuneus and the inferior parietal cortex (IPC) (Northoff et al., 2006; Van Buuren et al., 2010). Recent

${ }^{1}$ University of Helsinki and Helsinki University Hospital, Psychiatry, Helsinki, Finland

${ }^{2}$ Aalto NeuroImaging, Aalto University, Espoo, Finland

${ }^{3}$ Aalto University, School of Science, Department of Neuroscience and Biomedical Engineering, Espoo, Finland

4Turku PET Centre and Department of Psychology, University of Turku, Turku, Finland

5University of Helsinki, Institute of Behavioural Sciences, Helsinki, Finland

${ }^{6}$ Folkhälsan Research Center, Helsinki, Finland

${ }^{7}$ National Institute of Health and Welfare, Department of Mental

Health and Substance Abuse Services, Helsinki, Finland

${ }^{8}$ University Department of Psychiatry, 0xford, UK

${ }^{9}$ Vaasa Hospital District, Department of Psychiatry, Vaasa, Finland

\section{Corresponding author:}

Jesper Ekelund, Department of Psychiatry, University of Helsinki, P0 Box 590, FI-00029 HUS, Finland.

Email: jesper.ekelund@helsinki.fi 
research has emphasized the role of these regions in the pathogenesis of depression. Increased resting state metabolism and connectivity of the CMS, particularly the MPFC and ACC, have been repeatedly observed in depressed patients (Kaiser et al., 2015; Northoff, 2007), although decreased connectivity has also been reported (Anand et al., 2005; Phillips et al., 2015). Increased activity of the CMS, particularly the MPFC, during self-referential processing, as well as a diminished ability to deactivate these regions during emotional tasks, have also been found in depressed patients (Lemogne et al., 2012; Sheline et al., 2009). Further, some studies have shown that the alterations in the CMS activity are associated with increased self-focus and rumination as well as with higher levels of depression symptoms (Hamilton et al., 2011; Lemogne et al., 2012). An inability of the CMS to regulate self-referential activity could be essential in the pathogenesis of depression (Sheline et al., 2009).

Recently, there has been increasing interest in how antidepressants affect the processing of emotional information in the brain. Studying the effects of antidepressants in healthy subjects facilitates examining the direct actions of antidepressants without the confounding effects of depressed mood and mood improvement. Serotonergic and noradrenergic antidepressants have been shown to influence emotional processing by, for example, increasing recognition of happy facial expressions in healthy volunteers (Harmer et al., 2003a, b, 2008; Murphy et al., 2009), though not all the studies have been able to replicate this finding (Browning et al., 2007; Harmer et al., 2004). This effect is rapid, within hours or days from administration, despite the well-known delay in the clinical effect of antidepressants (Harmer and Cowen, 2013). In healthy volunteers, amygdala responses to negative visual stimuli seem to decrease as fast as a few hours or days after administration of several different antidepressants (Anderson et al., 2007; Del-Ben et al., 2005; Murphy et al., 2009; Norbury et al., 2007; Takahashi et al., 2005), even though increased amygdala responses (Bigos et al., 2008) or no effect at all (Norbury et al., 2007) have been reported in some studies. Recent meta-analysis also found both decreased and increased amygdala responses to negative stimuli after a single dose of antidepressant (Ma, 2014). These rapid changes in emotional processing are mostly opposite to the negative bias in information processing of depressed patients, and thus, antidepressants may be able to rapidly reverse this negative bias, which may be central to their antidepressant effect. However, although selfreferential processing is a core issue in pathogenesis of depression, the effects of antidepressants on self-referential processing remain elusive. A one-week administration of reboxetine was found to increase activity of fronto-parietal regions during categorization of positive self-referential words, and to decrease their activity during subsequent recognition of positive self-referential words (Norbury et al., 2008). The authors suggested this may indicate enhanced salience to positive cues and, consequently, reduced retrieval efforts for these words. A single dose of reboxetine, on the other hand, had no effect on neural responses to categorization of self-referential words. (Miskowiak et al., 2007). A three-week administration of escitalopram in healthy volunteers decreased responses of the PCC to self-referential words (Matthews et al., 2010).

Mirtazapine is a commonly-used antidepressant that has complex effects on serotonergic and noradrenergic transmission. It increases serotonin and norepinephrine release by blocking presynaptic alpha 2 receptors, and has additional antagonist action on $5 \mathrm{HT}_{2 \mathrm{~A}}, 5 \mathrm{HT}_{2 \mathrm{C}}$ and $5 \mathrm{HT}_{3}$ receptors (Fawcett and Barkin, 1998). It is also a high-affinity antagonist at the histamine H1 receptor (Fawcett and Barkin, 1998). The clinical profile of mirtazapine is different from that of SSRIs, as mirtazapine has an early anxiolytic effect (Fawcett and Barkin, 1998), possibly due to $5 \mathrm{HT}_{2 \mathrm{~A}}$ and $5 \mathrm{HT}_{2 \mathrm{C}}$ antagonism (Quesseveur et al., 2012), as well as $5 \mathrm{HT}_{3}$ (Olivier et al., 2000) and $\mathrm{H} 1$ blockage (Lader and Scotto, 1998). By contrast, SSRIs may increase anxiety and agitation in some patients at treatment initiation, and a similar early anxiogenic effect has been described in animal studies also (Handley, 1995). This may explain why a single dose of SSRI citalopram increases recognition of fearful facial expressions, and repeated administration decreases it (Browning et al., 2007; Harmer et al., 2004), whereas mirtazapine decreases threat processing immediately after a single dose (Arnone et al., 2009). This makes the early effects of mirtazapine easier to interpret, thus rendering mirtazapine an appealing agent for evaluating the effects of antidepressants on emotional processing. Rawlings et al. (2010) found decreased right amygdala-hippocampal and fronto-striatal activation compared with placebo after a single 15 $\mathrm{mg}$ dose of mirtazapine in response to fearful versus happy facial expressions. Another study noted increased activation after 30 $\mathrm{mg}$ of mirtazapine relative to placebo in the IPC in response to a reward task of financial gain (Vollm et al., 2006). Thus, mirtazapine may rapidly decrease neural responses to threatening stimuli and increase responses to positive or rewarding stimuli. However, it remains unknown whether/how mirtazapine influences selfreferential processing. Considering the importance of self-referential processing and the underlying brain network in depression, this could significantly help to elucidate the systems-level mechanisms of action of mirtazapine.

Here we investigated the effects of a single dose of mirtazapine on self-referential processing in healthy volunteers. We quantified the effect on positive and negative self-referential processing separately, as mirtazapine is known to have the abovementioned valence-specific effects on emotional processing. Based on previous evidence of the effect of mirtazapine on processing of emotional information (Arnone et al., 2009; Rawlings et al., 2010) and the effect of two other monoaminergic antidepressants, reboxetine (Miskowiak et al., 2007; Norbury et al., 2008) and escitalopram (Matthews et al., 2010), on self-referential processing, we hypothesized that mirtazapine would decrease responses of the CMS to negative self-referential processing and increase responses to positive self-referential processing.

\section{Methods and materials}

\section{Subjects}

The participants were 30 healthy, native Finnish-speaking, righthanded volunteers aged 18-35 years. They were recruited via advertisement for university students and word of mouth. The participants were screened with the Structured Clinical Interview for DSM-IV Axis I Disorders (First et al., 2002). Exclusion criteria included any current or life-time psychiatric disorder, current use of illicit drugs or excessive consumption of alcohol $(>24$ units/week for men and $>16$ units/week for women) and use of antidepressants, antipsychotic agents, mood stabilizers, systemic corticosteroids, beta blockers or benzodiazepines. The study was 
approved by the Ethics Committee of Helsinki and Uusimaa Hospital District and conducted in accordance with the ethical standards of this committee. Written informed consent was obtained from each participant.

The participants were allocated into two groups to either receive a single dose of mirtazapine $15 \mathrm{mg}$ two hours prior to the fMRI, or to be scanned without mirtazapine as a control group in an open-label design. The study was originally designed as a randomized, placebo-controlled, double-blind, parallel-group design with a $30 \mathrm{mg}$ dose of mirtazapine, but after scanning ten participants the double-blinding was deemed impossible due the sedative effect of mirtazapine. Consequently the blinding was in effect broken for both researcher and subject, and the study was re-designed as an open-label protocol with a smaller mirtazapine dose of $15 \mathrm{mg}$. During the open-label phase we had to exclude six participants from the mirtazapine group due to excessive sedation or sleeping during the fMRI (participant told that she/he had fallen asleep during the task, or was asleep after the task, or if there were more than $10 \%$ of un-responded items in the task). Data collection was continued until there were 15 subjects without excessive sedation in both groups (six male, 24 female, mean age 24 years, SD 3.72). Thus the final sample size for the fMRI analysis was 30 participants. However, in the behavioural tasks one subject from the drug group was excluded from the analysis of categorization accuracy and reaction times because of missing data due to a technical problem. One subject from the control group was excluded as an outlier due to extremely long reaction times ( $>3$ SD from the group mean), resulting in a final sample of 28 participants in these analyses. One subject from the mirtazapine group had to be excluded from the recall memory test due to missing data resulting in a final sample of 29 participants.

\section{Questionnaires}

Two hours before the fMRI, the participants completed questionnaires including the Beck Depression Inventory (BDI-II (Beck et al., 1961)), the Beck Anxiety Inventory (BAI (Beck et al., 1988)) and a questionnaire about current affective states using words adapted from Russell's circumplex model of affect (Russell, 1980) on a five-point Likert scale. The words formed a circumplex with two dimensions, valence and arousal: tired (väsynyt) and bored (pitkästynyt) (negative valence, low arousal), sad (surullinen) and miserable (onneton) (negative valence, neutral arousal), nervous (hermostunut) and anxious (ahdistunut) (negative valence, high arousal), active (aktiivinen) and aroused (vireä) (high arousal, neutral valence), excited (innostunut) and peppy

(pirteä) (positive valence, high arousal), cheerful (iloinen) and happy (onnellinen) (positive valence, neutral arousal), content (tyytyväinen) and calm (tyyni) (positive valence, low arousal) and tranquil (rauhallinen) and passive (passiivinen) (low arousal, neutral valence). The assessment of affective states was repeated right before the fMRI, two hours after the first assessment.

\section{Task and stimuli for fMRI}

During fMRI the participants completed an emotional categorization task (Miskowiak et al., 2007). The stimuli consisted of 60 adjectives describing 30 unequivocally positive (e.g. honest, reliable, sympathetic) and 30 unequivocally negative (e.g. irresponsible, selfish, lazy) personality features in Finnish and 20 neutral words, namely ten times 'left' (vasen) and ten times 'right' (oikea). The words were presented in an event-related design for one second in random order, with an inter-stimulus interval randomly varying between $5000 \mathrm{~ms}$ and $9500 \mathrm{~ms}$. The Presentation software (Neurobehavioral Systems Inc., Albany, USA) controlled the stimulus delivery. Total duration of the task was 11 minutes. The participants were asked to imagine overhearing two people talking about him or her using the word presented on the screen. They were asked to imagine how they would feel and accordingly categorize the words as either positive or negative as quickly and accurately as possible. The categorizing was done with a response key box with two keys on their right hand. They were asked to press the left button (index finger) if the word was positive (i.e. they would feel pleasant when being described with the word) and right button (middle finger) if the word was negative (i.e. they would feel unpleasant). When the word 'left' was presented, they were asked to press the left button and when the word 'right' was presented the right button.

After fMRI the participants completed a surprise memory test of the adjectives in the fMRI task. This took place approximately 1.5 hours after the categorization test because of other assessments unrelated to the present protocol. In the free recall task, the participants were asked to write down as many words as they could remember from the categorization task. After this, they completed a recognition memory task. Sixty adjectives from the categorization task (targets) and 60 new adjectives (distracters) were shown on a computer screen for one second in a random order. The participants were asked to respond with key presses as quickly and accurately as possible, indicating whether or not each word was presented in the categorization task. The next word appeared on the screen only after participants responded. The distracters were matched with the target words by length (mean of targets 9.1 characters, mean of distracters 9.0 characters, $t=0.36, p=0.719$ ), frequency (database of word frequencies in Finnish through WWW-Lemmie 2.0, which is a web-based tool for dynamic corpus work in the Language Bank of Finland, administered by CSC - IT Centre for Science Ltd in Espoo, Finland; mean of targets 53.6, mean of distracters 53.6, $t=0.002$, $p=0.998$ ) and imageability (rated by the research group similarly as in (Cortese and Fugett, 2004); mean of targets 2.3, mean of distracters $2.3, t=0.08, p=0.937)$.

\section{Statistical analysis of baseline characteristics, questionnaires and behavioural data}

Statistical analyses of baseline characteristics, questionnaires and behavioural data were performed with SPSS Statistics software, version 21 (IBM Corporation, Armonk, USA). We analysed the baseline characteristics (age and comprehensive school grade point average) using independent samples t-test. A non-parametric Mann-Whitney U-test was used for baseline questionnaires BDI and BAI because of their skewed distributions in the study sample. For the affective state questionnaire, we analysed each sector of the circumplex separately (negative affect (NA), positive affect (PA), negative affect with high arousal (NA-HA), 
positive affect with high arousal (PA-HA), negative affect with low arousal (NA-LA), positive affect with low arousal (PA-LA), high arousal (HA) and low arousal (LA)). Change in each sector was calculated separately for each subject by subtracting assessment at time 1 from assessment at time 2, and these differences were then compared between the two groups using an independent samples t-test. We additionally compared the single item of 'tiredness' assessed right before fMRI (i.e. assessment at time 2) between the groups to assess sedation. A Mann-Whitney U-test was used to analyse accuracy of categorizing positive and negative words and to compare the number of intrusions (incorrect answers) between the groups in the free recall test (because of the skewed distributions). A repeated measures ANOVA (group $\times$ valence) was used to compare the reaction times between the groups in the emotional categorization task and to compare the number of correct words in the free recall test. For the word recognition task, we first calculated the non-parametric discrimination index A' (Grier, 1971) to eliminate the effect of possible response biases. The discrimination index $\mathrm{A}^{\prime}$ varies typically between 0.5 (chance level, hits=false alarms) and 1 (perfect recognition, hits $=100 \%$, false alarms $=0 \%$ ). It was calculated using a formula: $\mathrm{A}^{\prime}=0.5+[(\mathrm{H}-\mathrm{FA})(1+\mathrm{H}-\mathrm{FA})] /[(4 \mathrm{H}(1-\mathrm{FA})]$, where $\mathrm{H}=$ hits/ targets and $\mathrm{FA}=$ false alarms/distractors. A repeated measures ANOVA (group $\times$ valence) was conducted to compare the performance of the two groups.

\section{fMRI acquisition and analysis}

The MRI was performed on a 3 T MAGNETOM Skyra wholebody scanner (Siemens Healthcare, Erlangen, Germany) at the Advanced Magnetic Imaging Centre, Aalto NeuroImaging, Aalto University School of Science, Espoo, Finland. The images were acquired with a T2*-weighted echo-planar imaging (EPI) sequence consisting of 33 slices (TR $1700 \mathrm{~ms}$, TE $24 \mathrm{~ms}$, FOV $202 \mathrm{~mm}$, flip angle $70^{\circ}$, voxel size $3 \times 3 \times 4 \mathrm{~mm}$, ascending interleaved acquisition with no gaps between slices). A total of 385 volumes were acquired, preceded by three dummy scans to avoid equilibration effects. T1-weighted structural images were acquired at a resolution of $1 \times 1 \times 1 \mathrm{~mm}$ (TR $2530 \mathrm{~mm}$, TE $3.3 \mathrm{~mm}$ ).

Functional MRI data were preprocessed and analysed with SPM8 software (http://www.fil.ion.ucl.ac.uk/spm/). EPI images were corrected for slice acquisition time, realigned to the first scan by rigid-body transformation to correct head movements, and co-registered to the individual's structural MRI. The anatomical image was then normalized to standard template (Montreal Neuroimaging Institute template) using SPM's unified segmentation/normalization algorithm (New Segment tool) (Ashburner and Friston, 2005), and the resulting deformation field was applied to the EPI images. Finally, EPI images were smoothed with Gaussian kernel of FWHM $8 \mathrm{~mm}$.

A random-effects model was implemented using a two-stage process. The first-level (within-subject) general linear model (GLM) included three explanatory variables - negative adjectives, positive adjectives and neutral words - and realignment parameters as effects of no interest to account for motion-related variance. A high-pass filter of $60 \mathrm{~s}$ and AR(1) modelling of temporal autocorrelation were applied. Individual contrast images were computed for the following contrasts: all adjectives $>$ neutral words, positive adjectives $>$ neutral words, negative adjectives $>$ neutral words, positive adjectives $>$ negative adjectives and negative adjectives $>$ positive adjectives. The second-level analysis used these images in a new GLM, which generated statistical images, that is, SPM t-maps. The statistical threshold was set at $p<0.05$, FDR corrected at cluster level (primary voxel-wise threshold at $p<0.05$ ). Cluster-extent based thresholding increases sensitivity and takes into account the spatial relationship of the activation foci with each other (Friston et al., 1994). However, it does not determine the statistical significance of an activation at a specific location in the cluster, and thus has low spatial specificity when the clusters are large (Woo et al., 2014).

To reveal the brain regions involved in self-referential processing, we first tested the aforementioned contrasts in the unmedicated control group only using one-sample t-test. In this analysis, the primary voxel-wise threshold was set at $p<0.01$, the FDR-corrected cluster-level threshold being the same $p<0.05$ as in all analyses. Next, the effect of mirtazapine was assessed by comparing responses in the drug group and the control group with independent samples t-test. We performed one further exploratory analysis for the regions with significant group differences in self-referential processing (all adjectives $>$ neutral words) by extracting signal change (parameter estimates) for all stimulus types relative to baseline fixation (positive adjectives $>$ baseline, negative adjectives $>$ baseline, neutral words $>$ baseline) from this cluster and comparing the groups using independent samples t-test.

Finally, we determined whether the sedative effect of mirtazapine could confound the between-group comparisons. We added the participants' self-assessment of tiredness (right before fMRI, i.e. assessment 2) as a covariate in the second level model (one-sample t-test) of the drug group separately. If there is any strong correlation between sedation level and fMRI-responses, adding the covariate to the one-sample t-test in the medication group should diminish the responses markedly. We also performed additional analyses of group differences, including tiredness assessment as a general covariate.

\section{Results}

\section{Baseline characteristics and questionnaires}

No significant differences emerged between the groups in age, comprehensive school grade point average, BDI, BAI $(p>0.05$ in all, see Table 1$)$ or gender ( $3 / 15$ male in both groups). In the affective state questionnaire (Table 2), there was a significant difference between the groups in change (difference between assessment 1 and assessment 2) in NA-LA (mean 2.00/0.43, mirtazapine/control, $t=4.09, p=0.001$ ), PA-HA (mean $-1.20 / 0$, mirtazapine/control, $t=-2.23, p=0.034$ ), HA (mean $-1.67 /-029$, mirtazapine/ control, $t=-2.10, p=0.045$ ) and LA (mean 0.93/-0.07, mirtazapine/control, $t=2.08, p=0.048$ ). There was a significant difference between the groups in subjective tiredness assessed right before fMRI (i.e. assessment at time 2, mean 3.20/1.93 mirtazapine/control, $t=3.40, p=0.002$ ).

\section{Behavioural tasks}

No significant differences were present between the groups in accuracy of categorizing positive or negative personality trait 
words (Table 3 ). In repeated measures ANOVA (group $\times$ valence), both groups had significantly faster reaction times for positive than negative words (effect of valence $\mathrm{F}(1,26)=7.26, p=0.012$ ), but no main effect of group or valence $\times$ group interaction was found (Table 4). In the free recall task, the control group recalled more positive and negative words than the mirtazapine group

Table 1. Demographic characteristics and baseline ratings of mood and anxiety.

\begin{tabular}{llll}
\hline & $\begin{array}{l}\text { Mean } \\
\text { mirtazapine }\end{array}$ & $\begin{array}{l}\text { Mean } \\
\text { control }\end{array}$ & $t$ value $(p)$ \\
\hline Age & 23.5 & 23.8 & $-0.22(0.825)$ \\
$\begin{array}{l}\text { Grade point } \\
\text { average }\end{array}$ & 9.0 & 9.2 & $-1.52(0.140)$ \\
\hline & & & \\
& Median & Median & p (Mann-Whitney \\
& mirtazapine & control & U-test) \\
\hline BDI & 0.0 & 1.0 & 0.132 \\
BAI & 2.0 & 3.0 & 0.239 \\
\hline
\end{tabular}

BDI: Beck Depression Inventory; BAI: Beck Anxiety Inventory.

Table 2. Differences between assessment 2 and assessment 1 in each dimension of circumplex of the affective state questionnaire.

\begin{tabular}{llrr}
\hline & $\begin{array}{l}\text { Mean } \\
\text { mirtazapine }\end{array}$ & \multicolumn{1}{l}{$\begin{array}{l}\text { Mean control } \\
(\text { SD })\end{array}$} & \multicolumn{1}{l}{$t$ value $(p)$} \\
& $(S D)$ & & \\
\hline PA change & $-0.40(0.51)$ & $-0.64(1.08)$ & $0.76(0.454)$ \\
NA change & $0(0.38)$ & $-0.07(0.83)$ & $0.30(0.765)$ \\
PA-HA change & $-1.20(1.42)$ & $0(1.47)$ & $-2.23(0.034)$ \\
PA-LA change & $-0.27(0.88)$ & $-0.14(0.77)$ & $-0.40(0.692)$ \\
NA-LA change & $2.00(1.96)$ & $-0.43(1.16)$ & $4.09(0.001)$ \\
NA-HA change & $-0.20(0.68)$ & $0.36(0.84)$ & $-1.97(0.059)$ \\
LA change & $0.93(1.28)$ & $-0.07(1.33)$ & $2.08(0.048)$ \\
HA change & $-1.67(1.84)$ & $-0.29(1.64)$ & $-2.10(0.045)$ \\
\hline
\end{tabular}

PA: positive affect; NA: negative affect; LA: low arousal; HA: high arousal.
Table 3. Categorization accuracy (proportion of correct responses) for the mirtazapine and control groups in the emotional categorization task.

\begin{tabular}{llcll}
\hline & & $\begin{array}{l}\text { Median } \\
\text { mirtazapine }\end{array}$ & $\begin{array}{l}\text { Median } \\
\text { control }\end{array}$ & $\begin{array}{l}p \text { (Mann-Whitney } \\
\text { U-test) }\end{array}$ \\
\hline Categorization & Positive & 97 & 100 & 0.178 \\
accuracy \% & Negative & 100 & 100 & 0.356 \\
\hline
\end{tabular}

(main effect of group $\mathrm{F}(1,27)=5.52, p=0.026$, see Table 4). There was no significant difference in the number of incorrect answers between the groups. In the recognition memory task, the mirtazapine group performed worse than the control group, and the difference was significant in ANOVA (main effect of group $\mathrm{F}(1,28)=9.67, p=0.004)$. No difference was found in performance between positive and negative words either within or between groups.

\section{fMRI results}

Self-referential processing in the control group. Self-referential processing (all adjectives $>$ neutral words) was associated with stronger activation of bilateral DMPFC and VMPFC extending to the ventral ACC, left PCC, left ventrolateral prefrontal cortex and orbitofrontal cortex (left orbitofrontal gyrus and inferior frontal gyrus), left hippocampus, left temporal cortex (left temporal pole, also a more posterior part of middle and inferior temporal gyri), left IPC (angular gyrus), bilateral occipital cortex (cuneus, lingual gyrus, fusiform gyrus) and cerebellum (Figure 1).

Negative self-referential processing (negative adjectives $>$ neutral words) increased activation of the same regions. However, the IPC was not activated in this contrast. Positive self-referential processing (positive adjectives $>$ neutral words) also activated similar regions and, additionally, the right amygdala. The contrast 'negative>positive adjectives' activated the left anterior insula and striatum (putamen and pallidum). No significant activation was found in the contrast 'positive $>$ negative adjectives'. However, with a more lenient primary voxel-wise threshold of $p<0.05$ (cluster-level FDR-corrected threshold

Table 4. Performance in the emotional categorization and memory tasks for the mirtazapine and control groups.

\begin{tabular}{|c|c|c|c|c|c|c|c|c|c|c|c|}
\hline & & \multicolumn{2}{|c|}{ Mirtazapine } & \multicolumn{2}{|l|}{ Control } & \multicolumn{2}{|c|}{ Main effect of valence } & \multicolumn{2}{|c|}{ Main effect of group } & \multicolumn{2}{|l|}{ Interaction } \\
\hline & & Mean & SD & Mean & SD & $\mathrm{F}$ & $p$ & $\mathrm{~F}$ & $p$ & $\mathrm{~F}$ & $p$ \\
\hline \multirow{2}{*}{$\begin{array}{l}\text { Categorization } \\
\text { response times, } \\
\text { ms }\end{array}$} & Positive & 963.9 & 264.29 & 969.6 & 256.32 & $F(1,26)=7.256$ & 0.012 & $F(1,26)=0.018$ & 0.894 & $F(1,26)=0.588$ & 0.450 \\
\hline & Negative & 1054.0 & 362.68 & 1019.8 & 259.76 & & & & & & \\
\hline \multirow{2}{*}{$\begin{array}{l}\text { Free recall } \\
\text { correct responses }\end{array}$} & Positive & 3.36 & 2.34 & 5.33 & 2.64 & $F(1,27)=1.114$ & 0.301 & $F(1,27)=5.524$ & 0.026 & $F(1,27)=0.203$ & 0.656 \\
\hline & Negative & 3.14 & 2.03 & 4.80 & 2.08 & & & & & & \\
\hline \multicolumn{12}{|l|}{ Recognition } \\
\hline \multicolumn{12}{|l|}{ accuracy } \\
\hline \multirow[t]{2}{*}{ hits/targets $\%$} & Positive & 60.00 & 19.19 & 67.56 & 14.06 & & & & & & \\
\hline & Negative & 52.67 & 17.82 & 60.67 & 20.05 & & & & & & \\
\hline \multirow{2}{*}{$\begin{array}{l}\text { false alarms/ } \\
\text { distractors \% }\end{array}$} & Positive & 35.40 & 15.38 & 21.38 & 12.92 & & & & & & \\
\hline & Negative & 22.89 & 13.62 & 17.78 & 10.74 & & & & & & \\
\hline \multirow[t]{2}{*}{$A^{\prime}$} & Positive & 0.68 & 0.13 & 0.82 & 0.07 & $F(1,28)=1.091$ & 0.305 & $F(1,28)=9.669$ & 0.004 & $F(1,28)=2.926$ & 0.098 \\
\hline & Negative & 0.73 & 0.12 & 0.81 & 0.09 & & & & & & \\
\hline
\end{tabular}


$p<0.05$ ), we observed increased responses of the MPFC, ACC, MCC and PCC, as well as the bilateral amygdala-hippocampal complex and thalamus to positive as compared to negative selfreferential words.

Effects of mirtazapine. Mirtazapine decreased responses of the bilateral DMPFC, right VMPFC and right ventral ACC (Figure 2, Table 5) to self-referential processing (all adjectives $>$ neutral words). No significant differences were found between the mirtazapine and control groups in negative self-referential processing (negative adjectives $>$ neutral

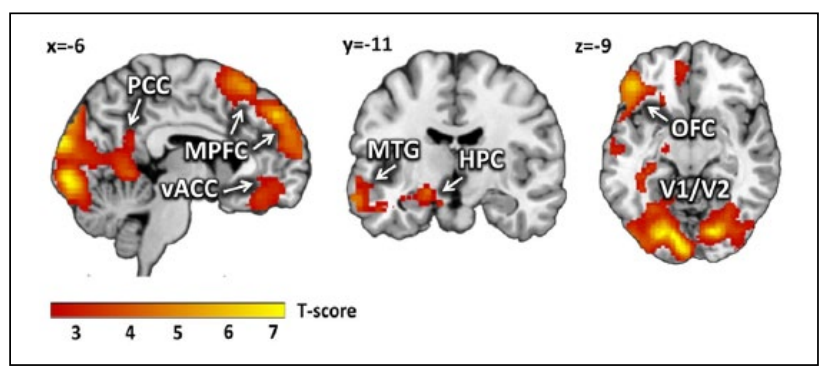

Figure 1. Brain regions with significantly increased responses to self-referential processing (all adjectives $>$ neutral words) in the un-medicated control group $(p<0.05$, FDR corrected at cluster level (primary voxel-wise $p<0.01)$ ). HPC: hippocampus; MPFC: medial prefrontal cortex; MTG: medial temporal gyrus; OFC: orbitofrontal cortex; PCC: posterior cingulate cortex; VACC: ventral anterior cingulate cortex; V1/V2: primary and secondary visual cortex. words). However, we observed a trend towards decreased responses of the DMPFC in the mirtazapine group (uncorrected cluster-level $p=0.008$ ). Mirtazapine decreased responses to positive self-referential processing (positive adjectives $>$ neutral words) in the bilateral anterior CMS including the DMPFC, VMPFC and ventral ACC, the parietal cortex including left IPC (angular gyrus), the bilateral PCC and precuneus and the occipital cortex including the primary (cuneus) and secondary (lingual gyrus) visual cortices (Figure 3, Table 5). When comparing positive versus negative self-referential processing (positive adjectives $>$ negative adjectives), mirtazapine was associated with decreased responses of the bilateral PCC and precuneus, the bilateral hippocampus, the parahippocampal gyrus and amygdala, the left anterior and posterior parts of temporal cortex (middle temporal gyrus) and the left fusiform gyrus (Table 5). No significantly decreased responses were found when comparing negative versus positive self-referential processing (negative adjectives $>$ positive adjectives). Adding subjective sedation (tiredness) as a covariate in the mirtazapine group, one-sample t-test of each contrast did not essentially change the results. There was no significant main effect of tiredness. Adding tiredness as a covariate in the analysis of group differences did not weaken the results.

In the complementary region-of-interest analysis of signal change, we found decreased responses to both positive and negative self-referential adjectives in the anterior CMS cluster (Figure 2, Table 6) in the mirtazapine group relative to the control group. Importantly, no significant differences were seen in responses to neutral words between the groups.

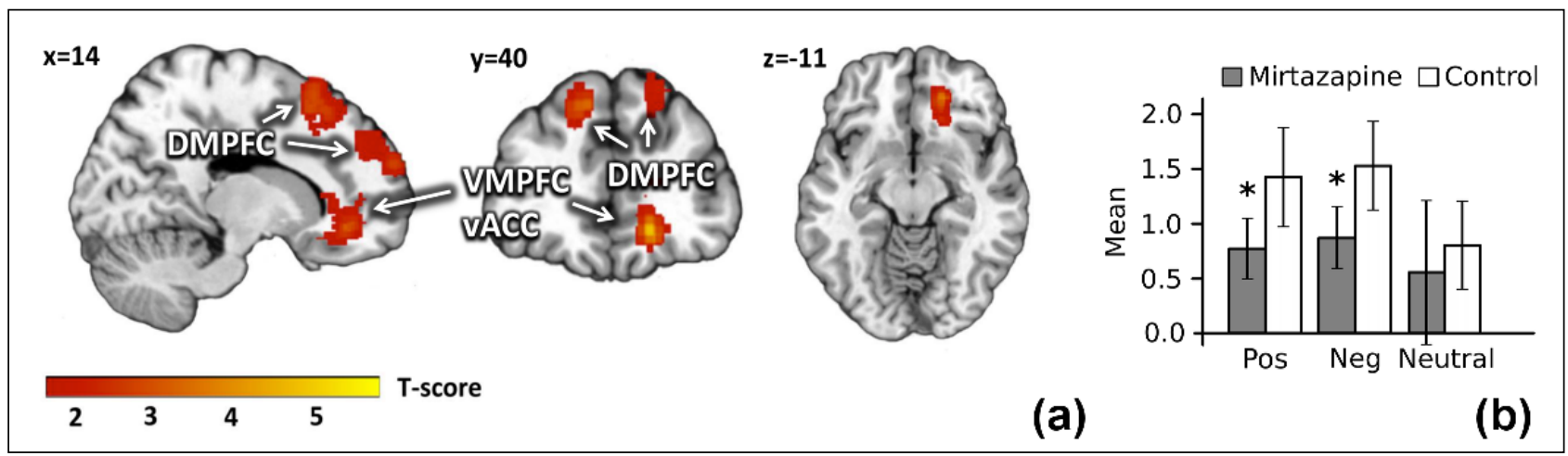

Figure 2. (a) Brain regions with significantly decreased responses to self-referential processing (all adjectives>neutral) in the mirtazapine group relative to the control group ( $p<0.05$, FDR corrected at cluster level). (b) Plot of mean signal change (parameter estimates) extracted from the significant cluster for all stimulus types relative to baseline fixation. Error bars represent standard error of mean. ${ }^{*} p<0.05$. DMPFC: dorsomedial prefrontal cortex; VMPFC: ventromedial prefrontal cortex; VACC: ventral anterior cingulate cortex.

Table 5. Peak activations of the clusters showing significant activation in the control group compared to mirtazapine group $(p<0.05$, FDR-corrected at cluster level).

\begin{tabular}{llllr}
\hline Contrast & Region & $p$-value & $z$-value & Coordinates \\
\hline Self-referential>neutral & MPFC/ACC & 0.043 & 4.20 & $18,40,-8$ \\
Positive>neutral & MPFC/ACC & 0.001 & 4.07 & $18,42,-8$ \\
Positive>negative & occipital/parietal & 0.016 & 3.49 & $28,-52,8$ \\
& temporal/parietal & 0.001 & 3.62 & $-22,-40,-10$ \\
\hline
\end{tabular}

MNI coordinates refer to peak activation within each cluster. 


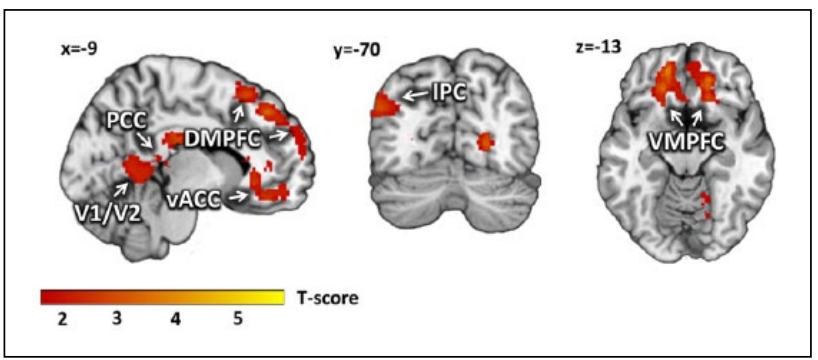

Figure 3. Brain regions with significantly decreased responses to positive self-referential processing (positive adjectives>neutral) in the mirtazapine group relative to the control group $(p<0.05$, FDR corrected at cluster level). DMPFC: dorsomedial prefrontal cortex; IPC: inferior parietal cortex; PCC: posterior cingulate cortex; VMPFC: ventromedial prefrontal cortex; $V A C C$ : ventral anterior cingulate cortex; V1/V2: primary and secondary visual cortex.

Table 6. Signal change (parameter estimates) extracted from the significant cluster in the medial prefrontal cortex and anterior cingulate cortex.

\begin{tabular}{llll}
\hline & $\begin{array}{l}\text { Mean } \\
\text { mirtazapine } \\
(\text { SD })\end{array}$ & $\begin{array}{l}\text { Mean } \\
\text { control } \\
(\text { SD })\end{array}$ & t value $(p)$ \\
\hline Positive>baseline & $0.77(0.54)$ & $1.42(0.89)$ & $-2.41(0.023)$ \\
Negative>baseline & $0.86(0.55)$ & $1.52(0.80)$ & $-2.61(0.014)$ \\
Neutral>baseline & $0.55(1.30)$ & $0.80(0.79)$ & $-0.64(0.530)$ \\
\hline
\end{tabular}

\section{Discussion}

We show for the first time that a single dose of mirtazapine influences self-referential processing in healthy volunteers. The results go significantly beyond the previous findings about the early effects of mirtazapine on sensory emotional processing (Arnone et al., 2009; Rawlings et al., 2010) and suggest that down-regulation of self-referential processing in the CMS may be a potential mechanism of action of antidepressants.

\section{Mirtazapine influences self-referential processing}

We found that self-referential processing of emotional adjectives activated the expected cortical midline areas (MPFC and ACC, PCC, precuneus) in non-medicated subjects. In line with previous studies, the activation foci extended to the left lateral prefrontal cortex and orbitofrontal cortex, left hippocampus, left temporo-parietal cortex, occipital cortex and cerebellum (Di Simplicio et al., 2012; Lane et al., 1997; Northoff, 2007; Van Buuren et al., 2010).

Our main finding was that mirtazapine decreases responses of the CMS to self-referential words at only two hours after a single dose. This is in accord with the previously reported early effect of two monoaminergic antidepressants, reboxetine and escitalopram, on self-referential processing of healthy volunteers (Matthews et al., 2010; Norbury et al., 2008). However, opposite to the effect of reboxetine reported previously, we found decreased responses, particularly to positive self-referential processing, whereas any decrease in responses to negative self-referential processing did not reach statistical significance. This seems counter-intuitive, and contrary to our expectations, as depressed patients are known to have a negative bias in information processing, and previous reports have shown that mirtazapine, as well as other antidepressants, is able to increase positive bias in healthy volunteers (Arnone et al., 2009; Norbury et al., 2008; Rawlings et al., 2010). However, our results in the post hoc analysis of signal change in the anterior CMS indicate that, in this area, activity was significantly decreased in the mirtazapine group relative to the control group in response to both positive and negative words. Acute versus chronic or subchronic administration may also be an important issue here; reboxetine was previously found to decrease responses to negative self-referential words after repeated administration, but not after a single dose (Miskowiak et al., 2007; Norbury et al., 2008). Interestingly, a recent meta-analysis also showed that acute effects of antidepressants on neural responses to negative stimuli seem to be both decreasing and increasing, but after repeated administration, consistently decreasing (Ma, 2014).

The tendency of healthy individuals to more likely evaluate positive information as self-descriptive (Moran et al., 2006), and increased responses of the CMS to positive vs negative self-referential processing found previously (Norbury et al., 2007) and here, may also influence our results. Mirtazapine may have a stronger attenuating effect on neural responses to positive adjectives because they are experienced as more self-related than negative adjectives. This remains, however, speculative, since we did not ask participants to provide evaluations about the self-relatedness of the words. The association of the valence of self-referential material with the modulating effect of mirtazapine might be different in depressed persons to healthy individuals. The attenuating effect of mirtazapine on positive self-referential processing could also reflect a general negative effect of serotonergic antidepressants on affective experience, which is sometimes reported (Price et al., 2009). This is supported by the mirtazapine-related decrease in positive affect-high arousal affective state and increase in negative affect-low arousal affective state observed here. However, this should be interpreted with caution, because the sedative effect of mirtazapine may alternatively explain the observed group differences in affective states, as we only found differences in positive and negative affect with the arousal dimension (including words 'peppy' and 'tired', respectively).

Studies consistently implicate the role of the CMS, especially the MPFC and ACC, in the pathogenesis of depression (Drevets et al., 2008). Further, a recent meta-analysis suggests that the midline structures such as the ACC, PCC, OFC and MPFC are affected by antidepressant treatment in response to emotional tasks in depressed patients and healthy subjects (Ma, 2014). Furthermore, the ACC appears to be a promising area to predict treatment response of depressed patients across different interventions, including antidepressant medication, transcranial magnetic stimulation and psychotherapy (Pizzagalli, 2011).The increased self-focus in depression is an important target of psychotherapy. For example, mindfulness-based cognitive therapy that specifically aims to increase awareness of and disengagement from negative self-focused thinking has been shown to prevent relapses of recurrent depression (Teasdale et al., 2000). It is possible that a down-regulated self-referential processing is essential for therapeutic effect across different treatment interventions, including antidepressant medication, and, especially, the anterior CMS may 
be an important neural substrate for this change. Mirtazapine can potentially influence the anterior CMS, as it is known to have a high binding potential in cortical regions (Smith et al., 2008) and the MPFC has a high density of particularly $5 \mathrm{HT}_{2 \mathrm{a}}$ receptors (Fisher et al., 2009).

The role of the anterior CMS in the pathogenesis of depression and the mechanisms of antidepressant treatments may be understood via their role in integrating information from the limbic, self-referential (CMS) and cognitive (including dorsolateral prefrontal cortex (DLPFC)) networks, all of which have been shown to be altered in depression (Kaiser et al., 2015; Lemogne et al., 2012). The anterior CMS are thought to act as a hub to link sensory, visceral and emotional signals to self-referential processes (Northoff, 2007; Northoff et al., 2006). The DLPFC is implicated in higher-order processing, which seems to be reciprocally linked to self-referential processing (Northoff et al., 2006). It has been argued that in depression hyperactivity of the anterior CMS and lack of inhibition from the DLPFC - that is, dominance of the self-referential network over the cognitive network - could lead to increased self-focus and rumination (Hamilton et al., 2011; Nejad et al., 2013). Overly-increased selffocus alongside and integrated with negative bias in information processing can form a vicious circle that maintains and reinforces depression (Northoff, 2007). Our results show that mirtazapine rapidly reduces responses of the CMS to self-referential information. This could lead to improved regulation of self-referential and emotional processing, which in depressed patients may result in decreased self-focus and rumination and improved ability to focus on the external world.

\section{Limitations}

We did not include a control condition with adjectives processed in a non-self-referential manner. This leaves open the possibility that the effect of mirtazapine observed here was due to emotional rather than self-referential content of the words; however, given the foci of the observed changes (the CMS rather than the limbic circuit), this does not seem likely. Using cluster-extent based thresholding hinders the spatial specificity of the results, as we found rather large significant clusters. However, even though the specific voxel-level location of the drug effect within the clusters remains elusive, the significant drug effects fall into the expected CMS.

We used an open-label design because the sedative effect of mirtazapine makes blinding practically impossible. Thus, the sedative effect of mirtazapine also complicates interpretation of the results, as it could be argued that the sedative effect is driving the differences between the groups in brain activation. This is unlikely for a number of reasons. First, adding the subjective tiredness as a covariate in the drug group model had essentially no influence on the responses of each event type. Furthermore, adding tiredness as a covariate in the analysis of group differences did not weaken the results. However, tiredness is not a direct measurement of sedation, and the lack of specific assessment of sedation should also be considered as a limitation of the study. Second, we did not find any differences in task reaction times between the two groups, indicating that any sedation in the drug group did not influence motor behaviour known to be sensitive to sedative effects (Kim et al., 2004). However, performance in the memory task was worse in the mirtazapine group. This may be related to sedation, but the memory effects of sedative drugs have also been argued to be a separate phenomenon from the sedation (Veselis et al., 2001; Wezenberg et al., 2007). The study sample was rather small and this makes it difficult to judge the clinical meaning of this secondary finding of reduced memory performance in the drug group. Third, we observed no between-group differences in neural responses to neutral words only. The selective effects of mirtazapine on emotional self-referential processing thus confirm that sedation does not play a major role in the observed findings.

It is also important to bear in mind that we studied acute effects of mirtazapine in healthy volunteers, and thus any interpretations about the long-term effects of mirtazapine, particularly in clinical populations, are speculative.

\section{Conclusion}

Mirtazapine decreases responses of the CMS to emotional selfreferential processing at only two hours after a single dose. These regions support self-referential processing and are implicated in the pathogenesis of depression. Our results suggest that mirtazapine can rapidly improve the ability of the CMS to down-regulate self-referential activity, which may lead to decreased self-focus and rumination, contributing to the antidepressant effect of mirtazapine. The results highlight the importance of the CMS and self-referential processing in the neuronal effects of antidepressants. Understanding these mechanisms can help to develop new treatment interventions specifically targeted at these mechanisms, and to predict the treatment response to various interventions.

\section{Acknowledgements}

We thank Marita Kattelus and other personnel of Aalto University Advanced Magnetic Imaging Centre for their help with the data acquisition.

\section{Declaration of Conflicting Interests}

The author CJH declared the following potential conflicts of interest with respect to the research, authorship, and/or publication of this article: $\mathrm{CJH}$ has received consultancy fees from Lundbeck, P1vital and Servier. She is a company director and shareholder of Oxford Psychologists Ltd. She holds current grant income from UCB and J\&J. All other authors declared no potential conflicts of interest with respect to the research, authorship, and/or publication of this article.

\section{Funding}

The authors disclosed receipt of the following financial support for the research, authorship, and/or publication of this article: This work was supported by the Academy of Finland (grant number 250935 and 265917).

\section{References}

Anand A, Li Y, Wang Y, et al. (2005) Activity and connectivity of brain mood regulating circuit in depression: A functional magnetic resonance study. Biol Psychiat 57: 1079-1088.

Anderson IM, Del-Ben CM, McKie S, et al. (2007) Citalopram modulation of neuronal responses to aversive face emotions: A functional MRI study. Neuroreport 18: 1351-1355.

Arnone D, Horder J, Cowen PJ, et al. (2009) Early effects of mirtazapine on emotional processing. Psychopharmacology (Berl) 203: 685-691. 
Ashburner J and Friston KJ (2005) Unified segmentation. Neuroimage 26: 839-851.

Beck AT, Epstein N, Brown G, et al. (1988) An inventory for measuring clinical anxiety: Psychometric properties. J Consult Clin Psychol 56: 893-897.

Beck AT, Ward CH, Mendelson M, et al. (1961) An inventory for measuring depression. Arch Gen Psychiatry 4: 561-571.

Bigos KL, Pollock BG, Aizenstein HJ, et al. (2008) Acute 5-HT reuptake blockade potentiates human amygdala reactivity. Neuropsychopharmacology 33: 3221-3225.

Browning M, Reid C, Cowen PJ, et al. (2007) A single dose of citalopram increases fear recognition in healthy subjects. $J$ Psychopharmacol 21: 684-690.

Cortese MJ and Fugett A (2004) Imageability ratings for 3000 monosyllabic words. Behav Res Methods Instrum Comput 36: 384-387.

Del-Ben CM, Deakin JF, McKie S, et al. (2005) The effect of citalopram pretreatment on neuronal responses to neuropsychological tasks in normal volunteers: An FMRI study. Neuropsychopharmacology 30: 1724-1734.

Di Simplicio M, Norbury R and Harmer CJ (2012) Short-term antidepressant administration reduces negative self-referential processing in the medial prefrontal cortex in subjects at risk for depression. $\mathrm{Mol}$ Psychiatry 17: 503-510.

Drevets WC, Price JL and Furey ML (2008) Brain structural and functional abnormalities in mood disorders: Implications for neurocircuitry models of depression. Brain Struct Funct 213: 93-118.

Fawcett J and Barkin RL (1998) Review of the results from clinical studies on the efficacy, safety and tolerability of mirtazapine for the treatment of patients with major depression. J Affect Disord 51: 267-285.

First MB, Spitzer RL, Gibbon M, et al. (2002) Structured clinical interview for DSM-IV-TR Axis I Disorders, Research Version, Patient Edition with Psychotic Screen. New York: Biometrics Research.

Fisher PM, Meltzer CC, Price JC, et al. (2009) Medial prefrontal cortex 5-HT(2A) density is correlated with amygdala reactivity, response habituation, and functional coupling. Cereb Cortex 19: 2499-2507.

Friston KJ, Worsley KJ, Frackowiak RSJ, et al. (1994) Assessing the significance of focal activations using their spatial extent. Hum Brain Mapp 1: 210-220.

Gotlib IH and Joormann J (2010) Cognition and depression: Current status and future directions. Ann Rev Clin Psychol 6: 285-312.

Grier JB (1971) Nonparametric indexes for sensitivity and bias: Computing formulas. Psychol Bull 75: 424-429.

Grunebaum MF, Keilp J, Li S, et al. (2005) Symptom components of standard depression scales and past suicidal behavior. $J$ Affect Disord 87: 73-82.

Hamilton JP, Furman DJ, Chang C, et al. (2011) Default-mode and task-positive network activity in major depressive disorder: Implications for adaptive and maladaptive rumination. Biol Psychiatry 70: $327-333$

Handley SL (1995) 5-hydroxytryptamine pathways in anxiety and its treatment. Pharmacol Therapeut 66: 103-148.

Harmer CJ, Bhagwagar Z, Perrett DI, et al. (2003a) Acute SSRI administration affects the processing of social cues in healthy volunteers. Neuropsychopharmacology 28: 148-152.

Harmer CJ and Cowen PJ (2013) 'It's the way that you look at it' - a cognitive neuropsychological account of SSRI action in depression. Phil Trans R Soc Lond B Biol Sci 368: 20120407.

Harmer CJ, Heinzen J, O'Sullivan U, et al. (2008) Dissociable effects of acute antidepressant drug administration on subjective and emotional processing measures in healthy volunteers. Psychopharmacology (Berl) 199: 495-502.

Harmer CJ, Hill SA, Taylor MJ, et al. (2003b) Toward a neuropsychological theory of antidepressant drug action: Increase in positive emotional bias after potentiation of norepinephrine activity. $\mathrm{Am} J$ Psychiatry 160: 990-992.
Harmer CJ, Shelley NC, Cowen PJ, et al. (2004) Increased positive versus negative affective perception and memory in healthy volunteers following selective serotonin and norepinephrine reuptake inhibition. Am J Psychiatry 161: 1256-1263.

Kaiser RH, Andrews-Hanna JR, Wager TD, et al. (2015) Largescale network dysfunction in major depressive disorder: A metaanalysis of resting-state functional connectivity. JAMA Psychiatry 72: 603-611.

Kim KM, Jeon WJ, Lee DH, et al. (2004) Changes in visual and auditory response time during conscious sedation with propofol. Acta Anaesthesiol Scand 48: 1033-1037.

Lader M and Scotto JC (1998) A multicentre double-blind comparison of hydroxyzine, buspirone and placebo in patients with generalized anxiety disorder. Psychopharmacology (Berl) 139: 402-406.

Lane RD, Fink GR, Chau PM, et al. (1997) Neural activation during selective attention to subjective emotional responses. Neuroreport 8: 3969-3972.

Lemogne C, Delaveau P, Freton M, et al. (2012) Medial prefrontal cortex and the self in major depression. $J$ Affect Disord 136: e1-e11.

Ma Y (2014) Neuropsychological mechanism underlying antidepressant effect: A systematic meta-analysis. Mol Psychiatry 20: 311-319.

Matthews SC, Simmons AN, Strigo IA, et al. (2010) Escitalopram attenuates posterior cingulate activity during self-evaluation in healthy volunteers. Psychiatry Res 182: 81-87.

Miskowiak K, Papadatou-Pastou M, Cowen PJ, et al. (2007) Single dose antidepressant administration modulates the neural processing of self-referent personality trait words. Neuroimage 37: 904-911.

Mor N and Winquist J (2002) Self-focused attention and negative affect: A meta-analysis. Psychol Bull 128: 638-662.

Moran JM, Macrae CN, Heatherton TF, et al. (2006) Neuroanatomical evidence for distinct cognitive and affective components of self. $J \operatorname{Cog} n$ Neurosci 18: 1586-1594.

Murphy SE, Norbury R, O’Sullivan U, et al. (2009) Effect of a single dose of citalopram on amygdala response to emotional faces. $\mathrm{Br} J$ Psychiatry 194: 535-540.

Nejad AB, Fossati P and Lemogne C. (2013) Self-referential processing, rumination, and cortical midline structures in major depression. Front Hum Neurosci 7: 666

Nolen-Hoeksema S (1991) Responses to depression and their effects on the duration of depressive episodes. J Abnormal Psychol 100: 569-582.

Norbury R, Mackay CE, Cowen PJ, et al. (2007) Short-term antidepressant treatment and facial processing. Functional magnetic resonance imaging study. Br J Psychiatry 190: 531-532.

Norbury R, Mackay CE, Cowen PJ, et al. (2008) The effects of reboxetine on emotional processing in healthy volunteers: An fMRI study. Mol Psychiatry 13: 1011-1020.

Northoff G (2007) Psychopathology and pathophysiology of the self in depression - neuropsychiatric hypothesis. J Affect Disord 104: $1-14$.

Northoff G, Heinzel A, de Greck M, et al. (2006) Self-referential processing in our brain - A meta-analysis of imaging studies on the self. Neuroimage 31: 440-457.

Olivier B, van Wijngaarden I and Soudijn W (2000) 5-HT3 receptor antagonists and anxiety; A preclinical and clinical review. Eur Neuropsychopharmacol 10: 77-95.

Phillips ML, Chase HW, Sheline YI, et al. (2015) Identifying predictors, moderators, and mediators of antidepressant response in major depressive disorder: Neuroimaging approaches. Am J Psychiatry 172: $124-138$

Pizzagalli DA (2011) Frontocingulate dysfunction in depression: Toward biomarkers of treatment response. Neuropsychopharmacology 36: $183-206$. 
Price J, Cole V and Goodwin GM (2009) Emotional side-effects of selective serotonin reuptake inhibitors: Qualitative study. Br J Psychiatry 195: 211-217.

Quesseveur G, Nguyen HT, Gardier AM, et al. (2012) 5-HT2 ligands in the treatment of anxiety and depression. Expert Opin Investig Drugs 21: $1701-1725$.

Rawlings NB, Norbury R, Cowen PJ, et al. (2010) A single dose of mirtazapine modulates neural responses to emotional faces in healthy people. Psychopharmacology (Berl) 212: 625-634.

Russell JA (1980) A circumplex model of affect. J Personality Soc Psychol 39: 1161-1178.

Sheline YI, Barch DM, Price JL, et al. (2009) The default mode network and self-referential processes in depression. Proc Nat Acad Sci USA 106: 1942-1947.

Smith DF, Hansen SB, Jakobsen S, et al. (2008) Neuroimaging of mirtazapine enantiomers in humans. Psychopharmacology (Berl) 200: 273-279.

Takahashi H, Yahata N, Koeda M, et al. (2005) Effects of dopaminergic and serotonergic manipulation on emotional processing: A pharmacological fMRI study. Neuroimage 27: 991-1001.
Teasdale JD, Segal ZV, Williams JM, et al. (2000) Prevention of relapse/ recurrence in major depression by mindfulness-based cognitive therapy. J Consult Clin Psychol 68: 615-623.

Van Buuren M, Gladwin TE, Zandbelt BB, et al. (2010) Reduced functional coupling in the default-mode network during self-referential processing. Hum Brain Mapp 31: 1117-1127.

Veselis RA, Reinsel RA and Feshchenko VA (2001) Drug-induced amnesia is a separate phenomenon from sedation: Electrophysiologic evidence. Anesthesiology 95: 896-907.

Wezenberg E, Sabbe BG, Hulstijn W, et al. (2007) The role of sedation tests in identifying sedative drug effects in healthy volunteers and their power to dissociate sedative-related impairments from memory dysfunctions. J Psychopharmacol 21: 579-587.

Vollm B, Richardson P, McKie S, et al. (2006) Serotonergic modulation of neuronal responses to behavioural inhibition and reinforcing stimuli: An fMRI study in healthy volunteers. Eur J Neurosci 23: $552-560$.

Woo C-W, Krishnan A and Wager TD (2014) Cluster-extent based thresholding in fMRI analyses: Pitfalls and recommendations. Neurolmage 91: 412-419. 